\title{
Study of complementary multi-sensors data influence on infrared thermography measurements for in-situ long-term monitoring
}

\author{
T. Toullier ${ }^{\mathrm{a}, \mathrm{b}}$, J. Dumoulin ${ }^{*}, \mathrm{~b}, \mathrm{a}$, and L. Mevel ${ }^{\mathrm{a}, \mathrm{b}}$ \\ anria, I4S Team, Campus de Beaulieu, 35042 Rennes, France \\ ${ }^{b}$ Ifsttar, COSYS-SII, Alle des Ponts et Chausses, 44344, Bouguenais, France
}

\begin{abstract}
Low cost solutions to infrastructure's thermal monitoring has become possible thanks to the development of uncooled infrared cameras over the past few years. However, in order to get a temperature value, one need to convert the radiative fluxes received by the camera. Such a step depends on spatial parameters, environment and the observed target characteristics which are usually unknowns or roughly estimated. To overcome this lack of knownledge, one solution is to combine the infrared camera measurements with other sensors. The purpose of this study is to examine the influence of the different parameters on the temperature conversion process and finally see to what extent complementary data can improve the estimation of the targeted object's temperature.
\end{abstract}

Keywords: infrared thermography, temperature, emissivity, calibration, data fusion, monitoring

\section{INTRODUCTION}

Latest improvements on uncooled infrared cameras have brought new opportunities for non-invasive diagnostics in the Civil Engineering's field. In the context of thermal monitoring, such sensor can provide an interesting option thanks to its full-field observation capability allowing to embrace large areas. However, retrieving with time over long period, current targeted object's apparent surface temperature from those measurements is a challenge that needs to be addressed with care ${ }^{1}$ In fact, the captured image depends on spatial parameters, the target's environment ${ }^{2 \sqrt{3}}$ and its thermo-physical properties ${ }^{4}$ that are usually unknowns $s^{\sqrt{5}}$ or roughly estimated . Part of a possible solution is therefore to use complementary environmental sensors in order to get a better estimatation of time present measurement environmental conditions that will have an influence on the apparent surface temperature reconstruction. The objective of this paper is to list and examine the influence of different parameters on the temperature conversion process through infrared thermography implemented in outdoor environmental conditions for long term thermal monitoring. Moreover, the possibility of improving the estimation of the targeted object's temperature through the fusion of multi-sensors data is considered. Thanks to the Cloud2IR acquisition system, ${ }^{6}$ an interesting amount of synchronized data have been gathered through years on various instrumented test sites, for instance: a small wood chalet in the framework of the SenseCity's experiments (IFSTTAR project). The temperature conversion model is improved by using meteorological reports (such as METAR) or in-situ data measurements acquired with various sensors such as pyranometers, pyrgeometers, weather stations, etc. In order to have proper acquisitions settings, the calibration process is also discussed. A radiative heat balance correction model for improving the temperature estimation through various sensors or local meteorological reports is firstly presented. Then, the tests sites involved in this study with their related instrumentation are presented. Finally, a summary of the sensors' data influence on the apparent temperature's scene estimation improvement, based on the availability and type of information is presented and discussed. Conclusion and perspectives are finally proposed.

* J.D.: E-mail: jean.dumoulin@ifsttar.fr, Telephone: +33 (0)2 40845998 


\section{TEMPERATURE'S DERIVATION FROM INFRARED MEASUREMENTS}

Temperature retrieval from infrared measurements in outdoor conditions on built or natural targets is facing, in many real situations, lack of knowledge about the observed surfaces and surrounding environment. The irradiance received at camera's sensor level is a combination of all the scene contribution as illustrated in Fig. 1. Therefore, one needs to gather information about the geometry of the scene, the meteorological conditions, and the radiative properties of the observed surfaces in order to retrieve the temperature from those measurements. Such approach can be achieved by implementing multiple sensors in-situ.

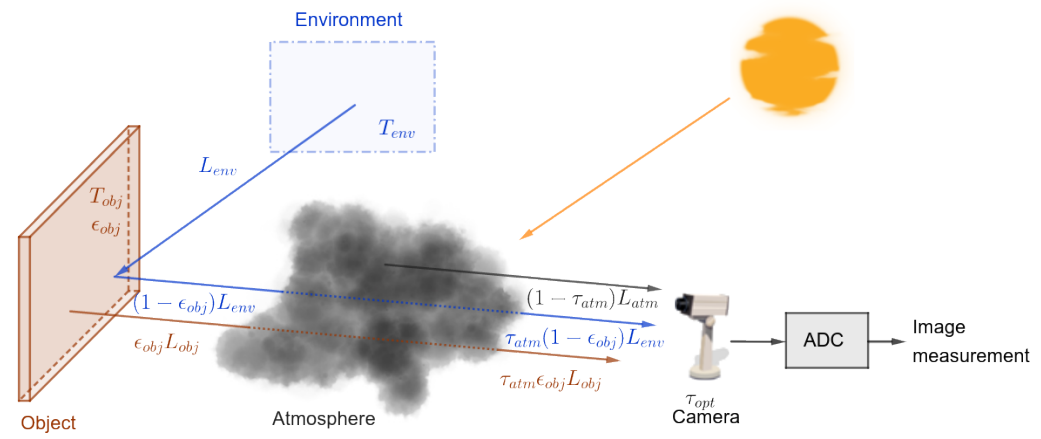

Figure 1: Radiation interactions during IR measurements

To convert the radiative flux received at the camera $L_{\text {total }}^{(i, j)}$ to temperatures, the following simplified radiometric model is used:

$$
L_{\text {total }}^{i, j}=\epsilon_{o b j} \tau_{a t m} \tau_{o p t} L_{o b j}^{i, j}+\left(1-\epsilon_{o b j}\right) \tau_{a t m} \tau_{o p t}\left(L_{e n v}^{i, j}+L_{\text {sun }}^{i, j}\right)+\left(1-\tau_{a t m}\right) \tau_{o p t} L_{a t m}+\left(1-\tau_{o p t}\right) L_{o p t}
$$

In outdoor conditions, environmental quantities can be derived from local weather station's data. The reflected, optics and atmospherics luminance values are computed by injecting a priori knowledge about the monitored objects, components of the infrared system and atmospherics conditions. Furthermore, a sky temperature correlation ${ }^{7}$ combined with inputs from in-situ solar and meteorological measurements can be used to estimate the reflected radiance contribution and atmospheric transmission, in particular when no reference targets can be implemented on real site. In other case, when data are missing, envornmental informations can be retrieved from meteorological aerodrome report (METAR) data, if available and by taking care of the scene geographical location.

In order to get consistent measurements, a thermal calibration is performed prior to the experiment in laboratory conditions. In the presented results, the calibration is based on the RBF-function (2), as described in 8 and derived from the Planck's law.

$$
T_{o b j}^{i, j}=\frac{B}{\log \left(\frac{R}{L_{o b j}^{i, j}}-F\right)}
$$

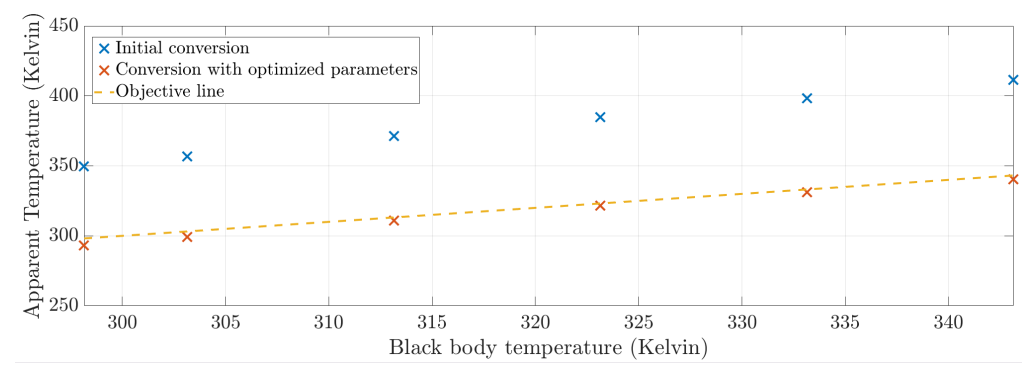

Figure 2: Optimized parameters results: the conversion is now near the objective line 
The result of such calibration is given Fig. 2, where the temperature values are compared with and without the optimized parameters. On this figure, the new parameters enable a more reliable conversion from the digital levels of the measured signal to the estimated temperature.

\section{EXPERIMENTAL SETUPS}

\subsection{Test site \#1: instrumented road section}

A road section that can be internally heated has been instrumented with different sensors. The monitoring is done with a FLIR SC655 thermal infrared camera $(640 \times 480$ LWIR FPA, pitch $17 \mu m$, and a $13.1 \mathrm{~mm}$ optical focal length). A weather station (Vaisala WXT 520), a net radiometer (Kipp \& Zonen CNR4) and a pyranometer (Kipp \& Zonen SPLite2) provides complementary measurements with meteorological data (rain, wind, temperature, etc.) and radiative heat fluxes. All those data are synchronized in time thanks to our multi-sensor data acquisition system Cloud2IR ${ }^{[6}$ Due to the particular angle of view, distance and planar properties of the scene, those data are interesting to process and analyse spatial sampling issues, mixed pixels and transmissions effects. Moreover, the presence of thermocouples on the road surface enables us some comparison with infrared measurements.

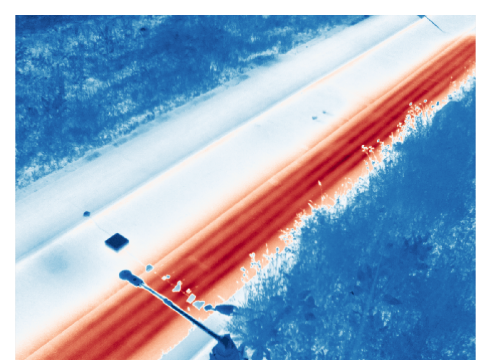

(a) Image of the road in the infrared spectrum

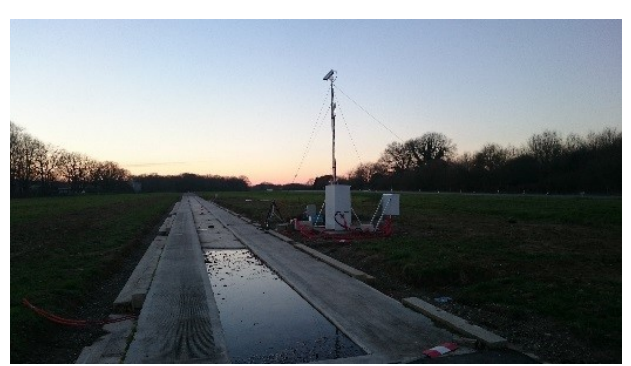

(b) Image of the road in the visible spectrum

Figure 3: Road section instrumentation: IR image (a) and test site overview (b)

\subsection{Test site \#2: instrumented wood house}

A wood chalet has been built and surveyed during 2 years in Paris under the Sense-City project experiments framework at IFSTTAR. It was monitored with a FLIR A65 thermal infrared camera $(640 \times 512$ LWIR FPA, pitch $17 \mu \mathrm{m}, 13 \mathrm{~mm}$ optical focal length), a weather station (Vaisala WXT 520) and a sunshine pyranometer (Delta-T SPN1) for the total and diffuse solar radiation. Cloud2IR was also used here for recording synchronized data $\underline{6}$

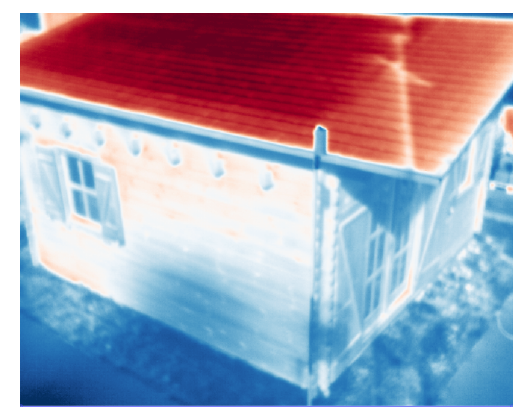

(a) Image of the chalet in the infrared spectrum

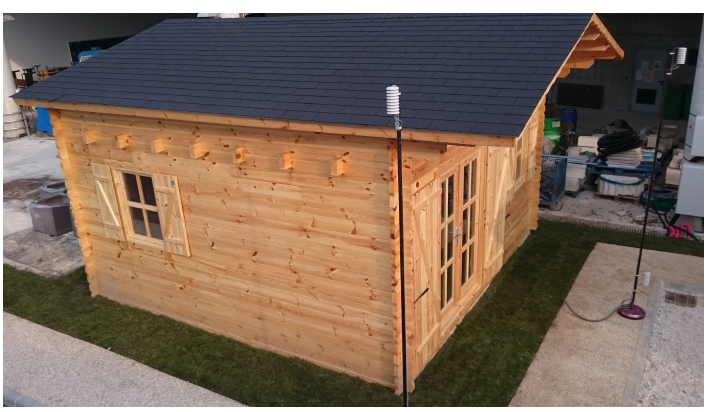

(b) Image of the chalet in the visible spectrum

Figure 4: Wood chalet survey instrumentation: IR image (a) and test site overview (b) 


\section{PARAMETER'S MODEL INFLUENCE ON TEMPERATURE ESTIMATION ERROR}

\subsection{Spatial considerations}

The positioning of the camera and the geometry of the scene will have an impact on the conversion process from digital levels recorded by the camera to the desired temperature. Nowadays, most infrastructures provides a BIM (Building Information Model) and in particular a 3D numerical model. In order to have knowledge on the actual camera view of the scene and thanks to this model, it is possible to retrieve the real world coordinates on the image, called resectioning (see Fig. 5p.

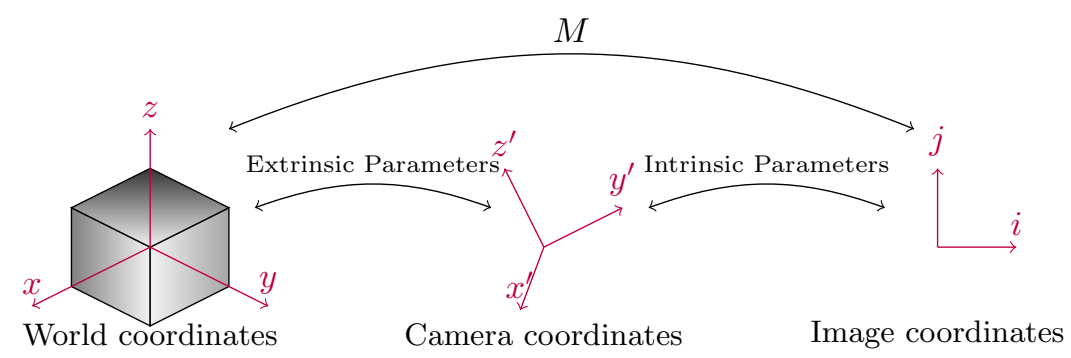

Figure 5: Resectioning principle, the conversion of the real world coordinates to the image ones

Let $M$ be a calibration matrix such as for every $(x, X) \in \mathbb{R}^{2} \times \mathbb{R}^{3}$ of coupled coordinates points between the image and the real world we have:

$$
x=M X
$$

By using the pin-hole model, we can define $M$ with optical and geometrical parameters:

$$
M=\left(\begin{array}{ccc}
f & s & c_{x} \\
0 & \text { af } & c_{y} \\
0 & 0 & 1
\end{array}\right)\left(\begin{array}{cccc}
1 & 0 & 0 & 0 \\
0 & 1 & 0 & 0 \\
0 & 0 & 1 & 0
\end{array}\right)\left(\begin{array}{cc}
R_{3 \times 3} & 0_{3 \times 1} \\
0_{1 \times 3} & 1
\end{array}\right)\left(\begin{array}{cc}
I_{3} & T_{3 \times 1} \\
0_{1 \times 3} & 1
\end{array}\right)
$$

Where those parameters are defined in Fig. 6. Since the system has 11 degrees of freedom, the knowledge of at least 6 couples of points $(x, X)$ is necessary. The Levenberg-Marquardt algorithm is finally used to solve the system.

\section{Extrinsics}

- Translation $T$ from the origin of world coordinates

- Rotation $R$ from the world coordinates

\section{Intrinsics}

- Focal length $f$

- Principle points $\left(c_{x}, c_{y}\right)$

- Aspect ratio a

- Skew $s$

Figure 6: Resectioning parameters with the pin-hole model

As exposed previously, the road section test site \#1 is mainly planar which is interesting for demonstrating the impact of the view angle to the conversion process. Once the resectioning has been applied, a distance map is obtained, representing the distance between the camera and the ground. In some cases, the model consideration is to use a constant distance over the entire image. However, the Fig. 7 shows this distance map (the range involved is $[5 ; 10]$ meters) and also the impact of the distance on the atmospheric transmission, for varying environmental conditions. On this figure, the impact of the distance can be quite important depending on the perspective involved. 


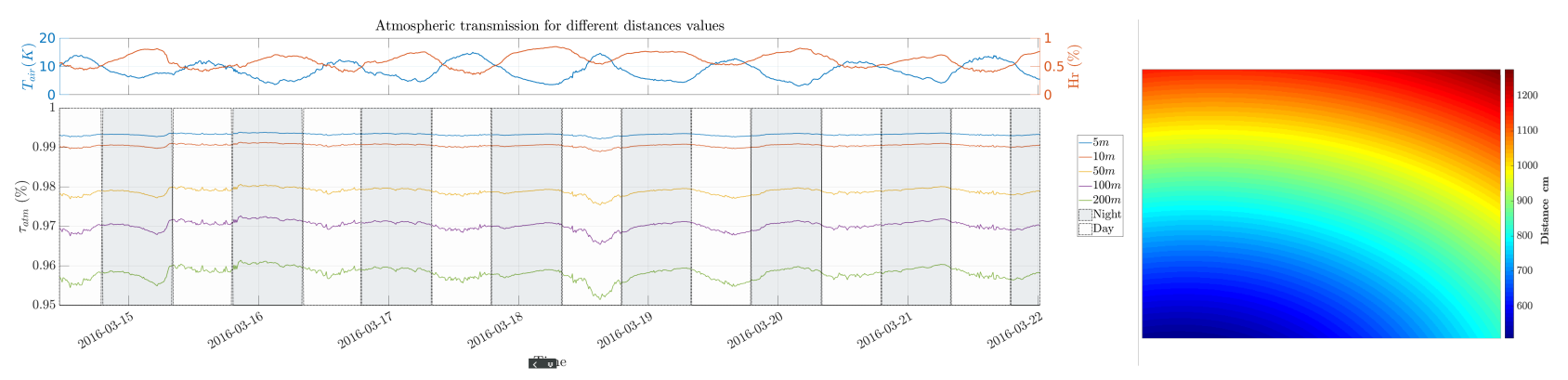

Figure 7: Impact of the distance on the atmospheric transmission and its evolution with time

Other spatial considerations must also be taken into account. Depending on the chosen optic and camera, images with varying spatial resolution and important angle between the observed surfaces and the camera may occur. In such cases, accurate temperature retrieval may be compromised due to non-square pixels, mixed pixels effects $\$$ and varying angle values along the same plane which may change the considered emissivity value. Moreover, when monitoring a particular plane or object in the scene, one may need to recover a given region of interest (ROI). The following figure shows an example of sampling issues due to a projection of a ROI on test site \#2 and an illustration of the mixed pixel effect where different materials' radiation are integrated over the same sensor.
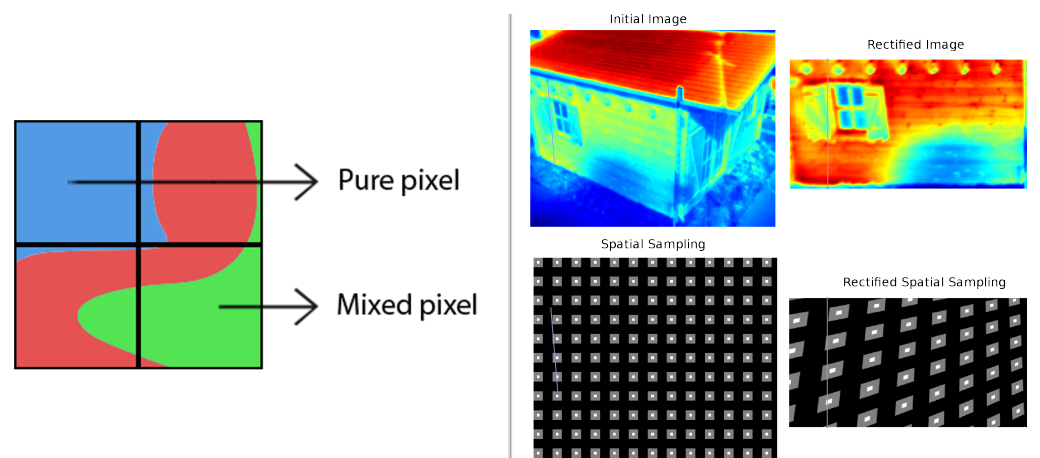

Figure 8: From left to right: Mixed pixel effect illustration. Spatial sampling issues due to reprojection

\subsection{Emissivity considerations}

In order to get information on thermo-optical wood house material's components and study their influence on apparent surface temperature estimation, emissivity measurements have been made on site with a portable measurement system, $\frac{10}{2}$ as shown in Table 1 and Fig. 9.

\begin{tabular}{llr} 
Material & Color & Emissivity \\
\hline Wood wall (west) & Blue & 0.95 \\
Wood wall (east) & Yellow & 0.90 \\
Grass & Green & 0.95 \\
Sidewalk & Red & 0.89 \\
Tar & Grey & 0.95 \\
Roof & Purple & 0.92
\end{tabular}

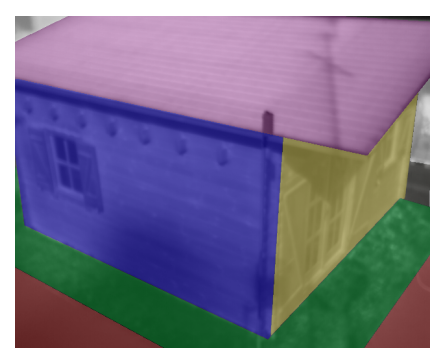

Table 1: Emissivity measurements values in the $7.5 \mu m-13 \mu m$ band.

Figure 9: Emissivity map for the wood house, colors represents one particular emissivity measurement 
Fig. 10 shows the effect of putting different emissivity values during the conversion of the digital levels to temperature. In the first case, a constant emissivity value is chosen over the entire image. In the middle image, the emissivity map represented in Fig. 9 is used in the conversion. The last image represents the difference between the two previous images.
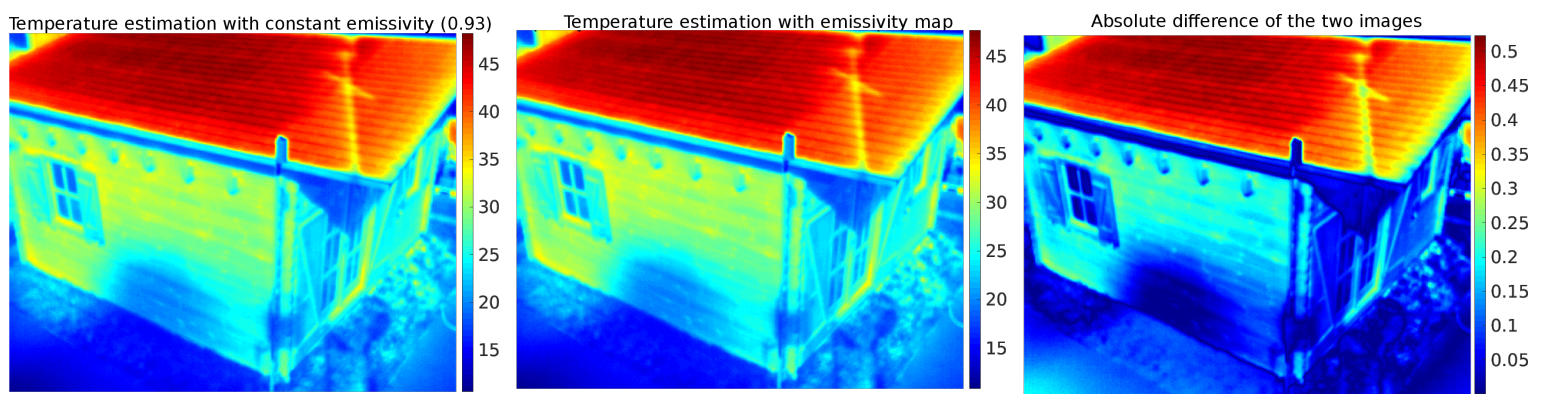

Figure 10: Emissivity impact on the conversion process

\subsection{Sky temperature considerations}

The sky temperature $\left(T_{s k y}\right)$ can be obtained by using in-situ measurements by using pyrgeometers data for example. In the following Fig. the temperature obtained from infrared data with sky correction and without sky correction is compared to the thermocouple's data at surface. Taking into account the sky temperature from local measurements gives a best estimate of the object temperature. The difference between the thermocouples measurements and the estimated temperature from infrared data with sky correction is then reduced.

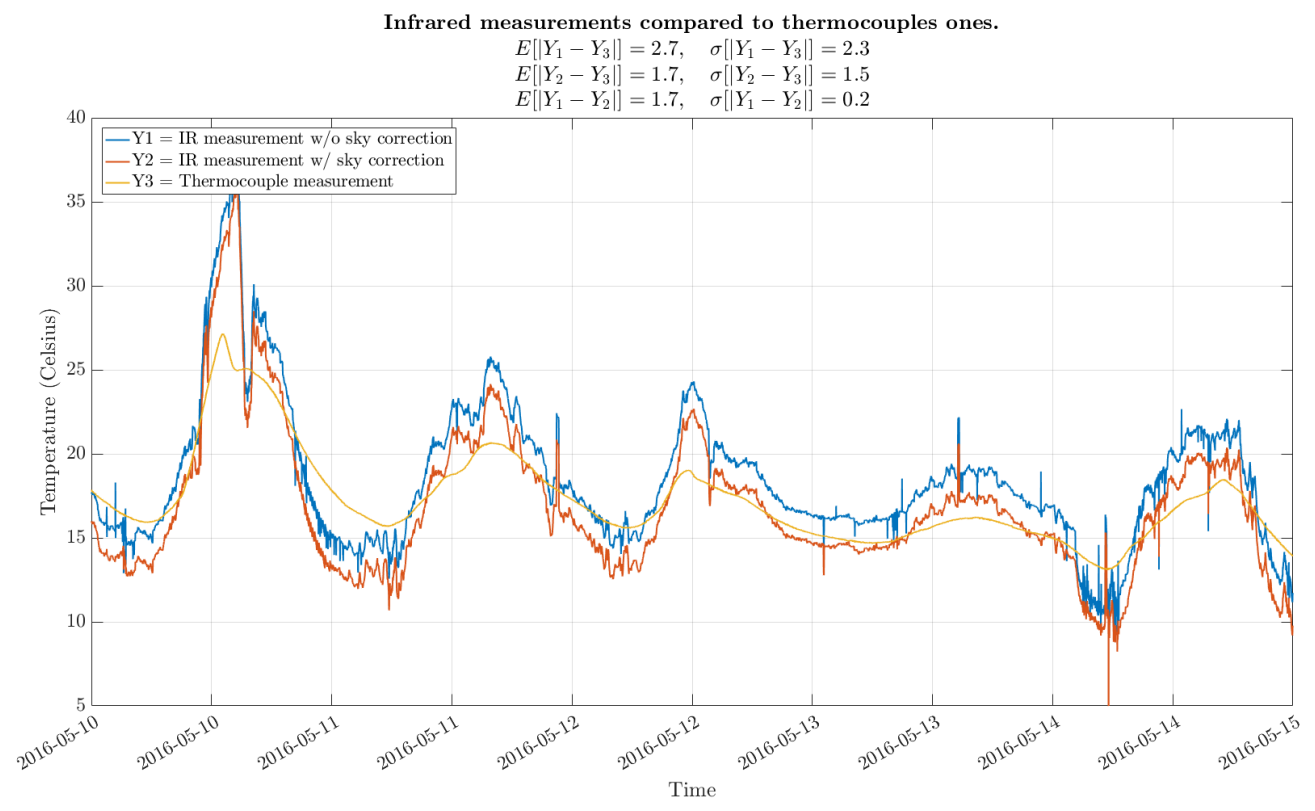

Figure 11: Thermocouples measurements compared to IR measurements temperature estimation with and without sky correction

When pyrgeometers data are not available, a possibility is to use a correlation from the literature that links the sky temperature with the air temperature, for example with the Swinbank's formula! ${ }^{[2]}$ The following figure shows the sky temperature derived from the Swinbank's when the air temperature data are obtained by three different ways:

- From the local weather station 
- From Copernicus' data store ${ }^{11}$

- From METAR data

Even if differences exists in term of sky temperature (see Fig. 12, it represents small radiation quantities regarding the involved temperature levels. Online data are therefore an interesting way to enrich the radiative model conversion when no local measurements are available.

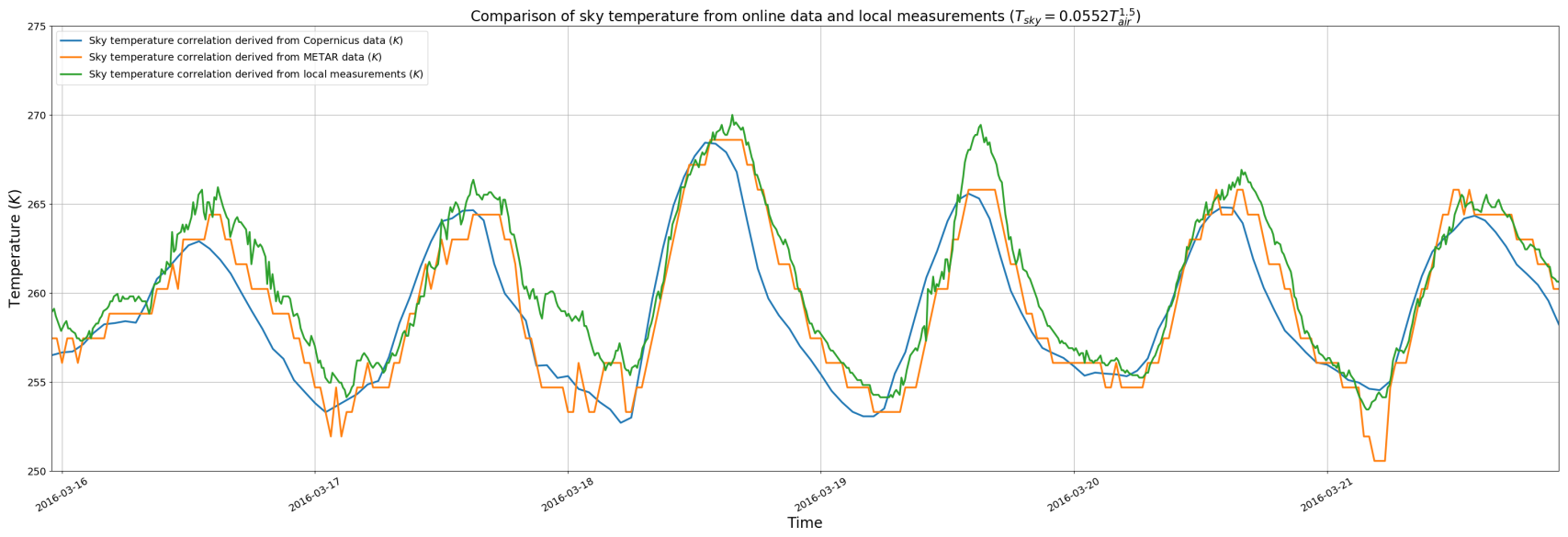

Figure 12: Sky temperature obtained with different air temperature data sources

In the following section, the sky temperature is derived from the Swinbanks correlation for two periods: in december and august. One can see that the sky temperature derived from the correlation will have an impact of apparent surface temperature images that depends on the period of observation for a same scene (here the wood chalet).

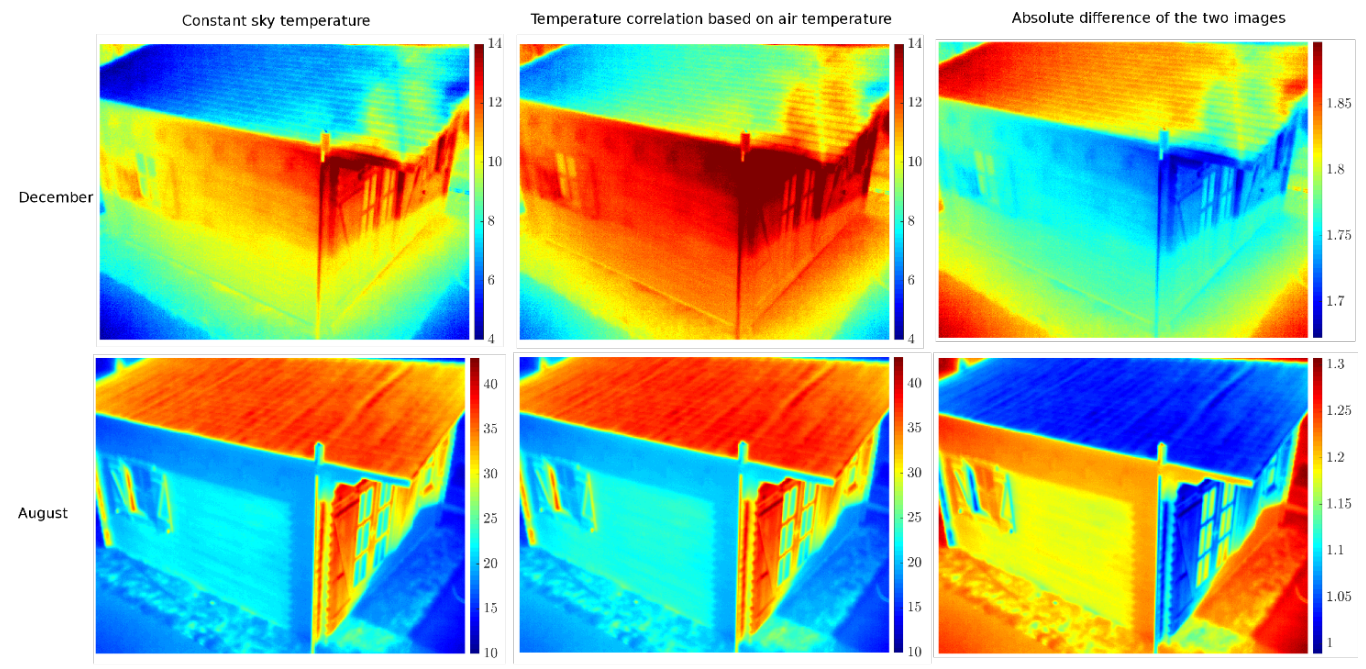

Figure 13: Swinbanks correlation for deriving the sky temperature

\subsection{Sun's irradiance considerations}

The sun's irradiance will also have an impact on the final temperature estimation. In fact, depending on the emissivity of the observed object, the irradiance at camera's sensor will contain the sun's reflected contribution, both specular and diffuse. An estimation of the solar irradiance can be obtained thanks to a pyrgeometer for 
example. In the case of the LWIR $(7.5 \mu m-13 \mu m)$, even though the sun contribution may be small compared to the NIR, SWIR and MWIR bands, it stills represents a source of error in the conversion process. In particular for low object's temperatures with lower emissivity as illustrated in Fig. 14.

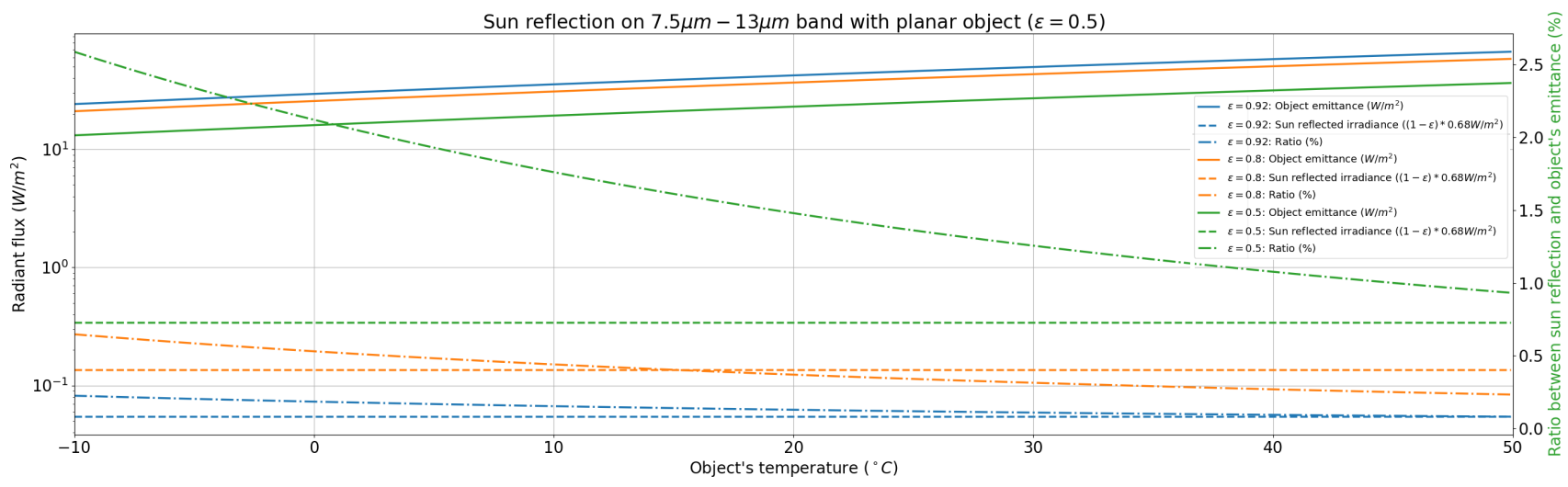

Figure 14: Sun irradiance reflection on $7.5 \mu m-13 \mu m$ band for a normal object with different emissivity values

We can see that the direct sun irradiance contribution can be responsible for up-to $2 \%$ of the object's emittance for a reflecting surface at $10^{\circ}$, leading to incorrect temperature estimation if not considered. Moreover, one can see that the error made on the temperature estimation will therefore vary depending on the time of the day. When local measurements are not available, one can use literature's models. The following figure shows the SMARTS2 $2^{12}$ irradiance's simulation compared to a local pyranometer measure in the SWIR band.

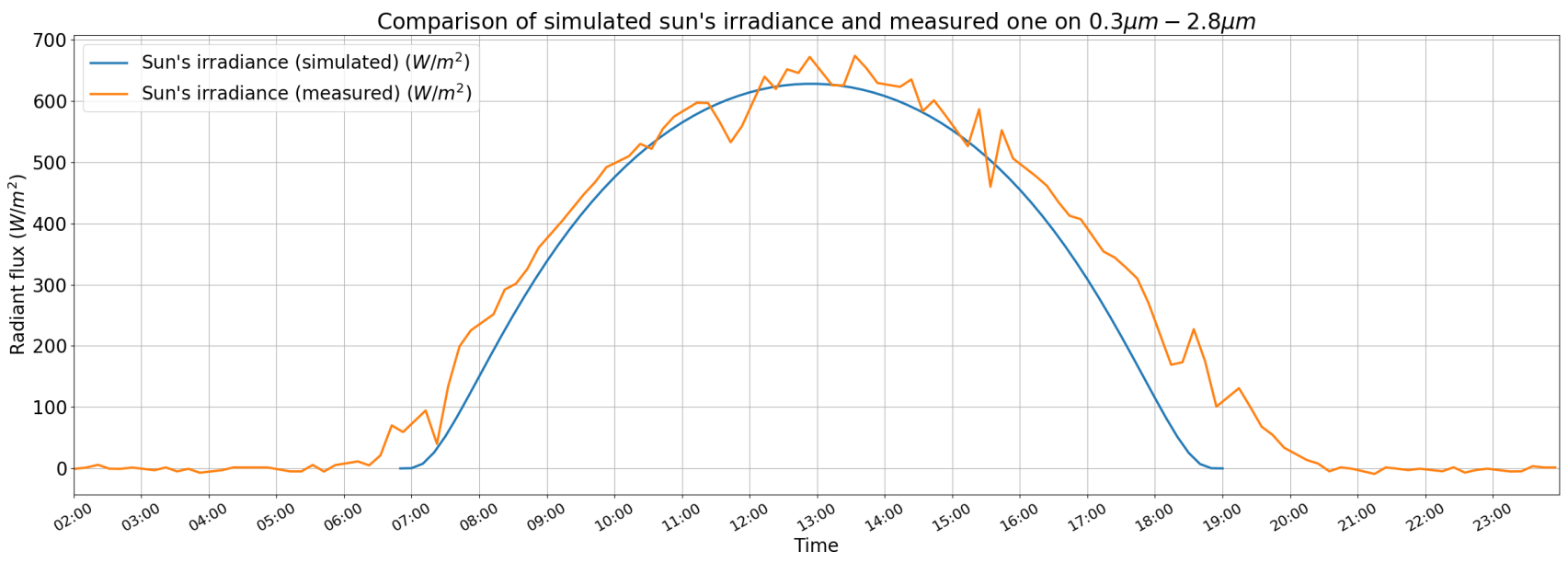

Figure 15: Comparison of sun's irradiance measured and simulated on may 5 th in the $0.3 \mu m-2.8 \mu m$ band

\subsection{Rain consideration}

The modification of emissivity due to fog or rain will also have an impact on the flux received at cameras sensors. In particular long-term measurement campaign involves variation of environment and climatic conditions (see Fig. 16. . Accurate temperature estimation is therefore difficult to obtain. However, the knowledge of meteorological conditions can lead to a post-process of the temperature estimation to warn about strong uncertainty assumption. To overcome this effect, a black-body source and an automatic thermal calibration triggered with the weather station rains' detection could be used. The radiative transmission model could be updated with a more proper one such as in! 13 


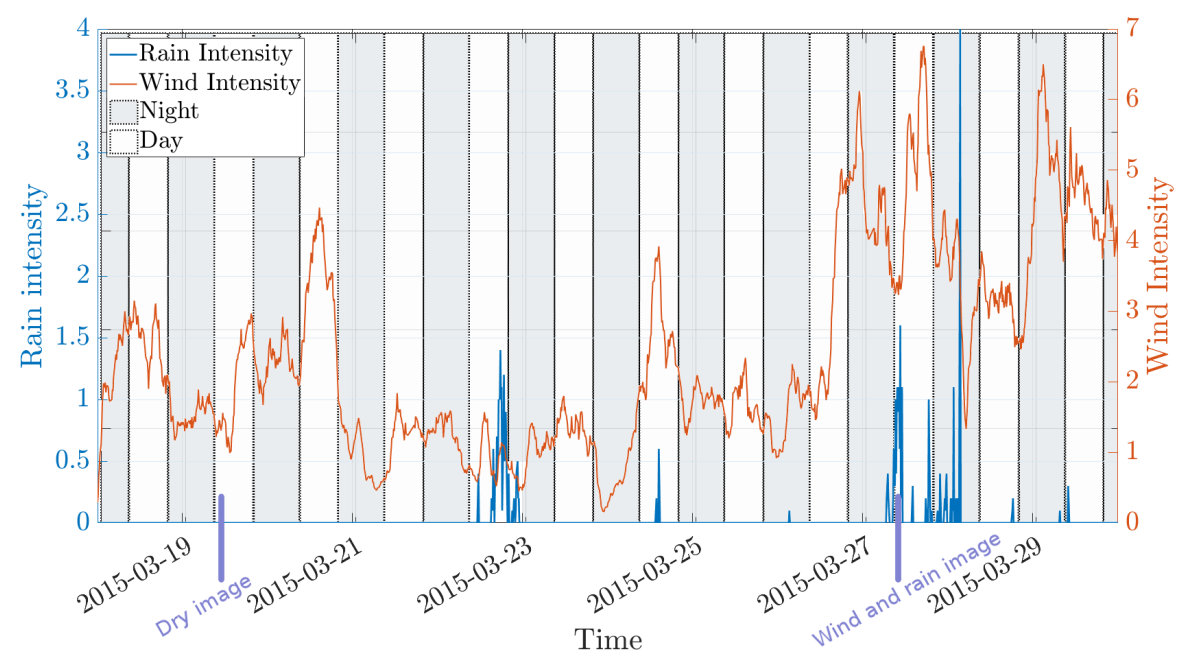

Figure 16: Rain episod issues for in-situ measurements

\subsection{Results}

The following figure represents the difference between a direct conversion of the digital levels to temperature by using the considerations presented previously in the radiative model or not over one chosen day.

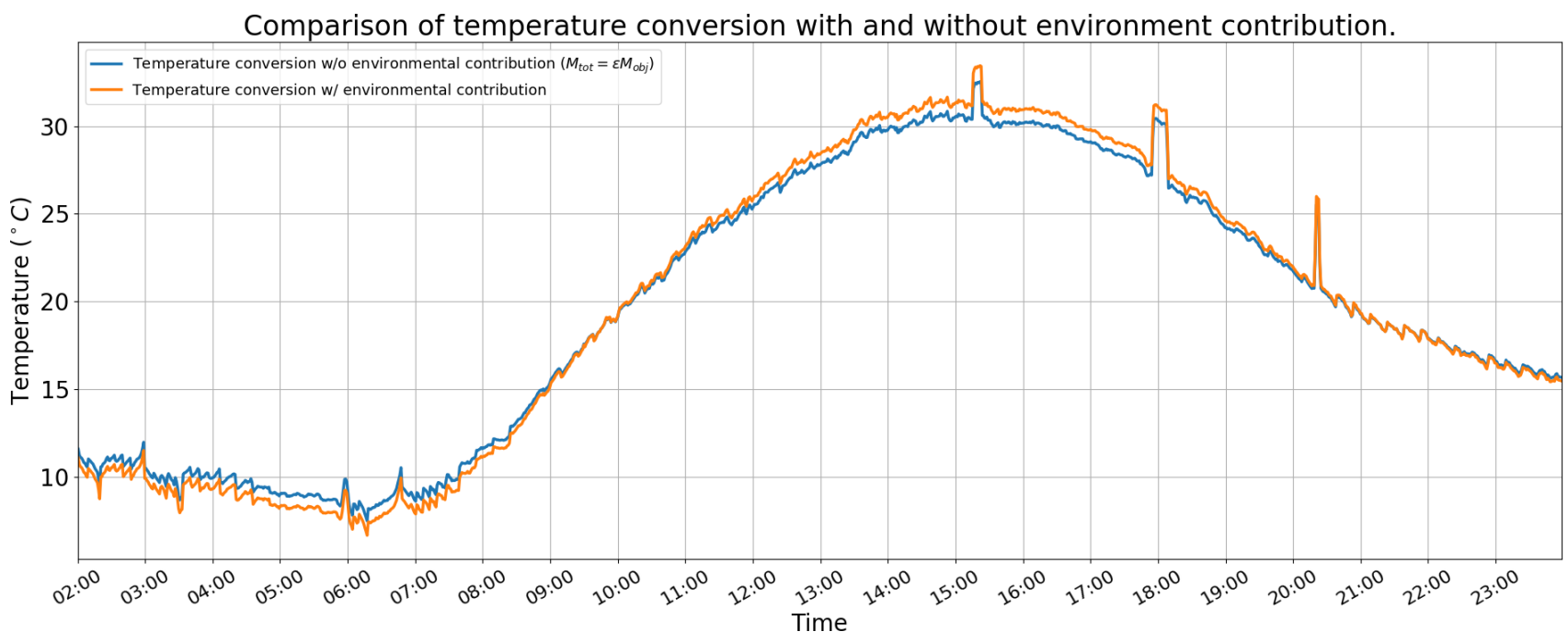

Figure 17: Comparison of temperature conversion with and without environment considerations

Fig. 17] shows that the error made by considering the environment contribution will be more or less important due to the fact that the errors come from different parameters that can compensate themselves. The peaks that we can observe in this figure represents the update of the camera's NUC (non-uniformity compensation) coefficients.

\section{CONCLUSION}

A review of different parameters involved into the long-term monitoring of structures through infrared thermography has been done. In such context, the temperature estimation for IR measurements needs to take into account many parameters or at least be aware of the involved difficulties to consider the estimation uncertainties. Using coupled measurements with those infrared data enables the temperature estimation to be more accurate. 
The spatial considerations in this study could be extended to a stereovision system which would bring more information on both geometric and optic properties, in particular to retrieve the depth of the image. Even if such algorithms have been used on the computer vision field, they need to be adapted for the infrared spectrum since the detector's responses are different. Selecting a region of interest in order to extract particular information within the image should be made cautiously. In fact, the sampling of the image due to the projection into the ROI may vary. Moreover, the mixed pixel effect as stated in this study should be more considered for accurate temperature measurement since IR thermography usually consider each pixel as a homogeneous material. Finally, considering the sky temperature, mapping the emissivity to the image materials and using a distance map reduces the difference between the temperature estimation and actual surfaces measurements. An angle map derived from the spatial resectioning and prior knowledge on the involved materials could improve the emissivity map. However, other considerations such as rain, sun's specular part, optics properties, lens flare, sensors' drifts, etc. could be incorporated more deeply into the radiative model. For example, thanks to the knowledge of the geometry of the scene and the time, the specular part of the sun could be derived. Again, if there is no sensor available, meteorological data available on the internet could be used. Even more, models such as MODTRAN or SMARTS2 for example could provide an estimation of the solar spectral irradiance at ground. Moreover, one of our perspective is to combine such radiative model with a Bayesian model to estimate conjointly emissivity and temperature.

\section{ACKNOWLEDGEMENTS}

Authors wish to thank for their support: French Region Bretagne and Sense-City Facility of Excellence of ANRs Future Investment Program.

\section{REFERENCES}

[1] Gaussorgues, G. and Chomet, S., [Infrared Thermography], Springer Science \& Business Media (Dec. 1993).

[2] Adelard, L., Pignolet-Tardan, F., Mara, T., Lauret, P., Garde, F., and Boyer, H., "Sky temperature modelisation and applications in building simulation," Renewable Energy 15, 418-430 (Sept. 1998).

[3] Dumoulin, J. and Boucher, V., "Infrared thermography system for transport infrastructures survey with inline local atmospheric parameter measurements and offline model for radiation attenuation evaluations," Journal of Applied Remote Sensing 8(1), 084978-084978 (2014).

[4] Howell, J., Siegel, R., and Pinar, M., [Thermal Radiation Heat Transfer.], CRC Press, 5th ed. (2010).

[5] Krapez, J.-C., "Radiative Measurements of Temperature," in [Thermal Measurements and Inverse Techniques], Heat Transfer, CRC Press, Boca Raton, FL (2011). OCLC: ocn587104377.

[6] Crinière, A., Dumoulin, J., and Mevel, L., "Management of local multi-sensors applied to SHM and longterm infrared monitoring: Cloud2IR implementation," Quantitative InfraRed Thermography Journal 0, 1-19 (Oct. 2018).

[7] Shettle, E. P. and Fenn, R. W., "Models for the aerosols of the lower atmosphere and the effects of humidity variations on their optical properties," tech. rep. (Sept. 1979).

[8] Toullier, T., Dumoulin, J., and Mevel, L., "Étude comparative de deux approches, thermocouples intégrés et thermographie infrarouge, pour la surveillance thermique d'une infrastructure de transport," in [25eme Congrès Français de Thermique], (2017).

[9] Jones, H. and Sirault, X., "Scaling of Thermal Images at Different Spatial Resolution: The Mixed Pixel Problem," Agronomy 4, 380-396 (July 2014).

[10] Monchau J-P, Marchetti M, I. L. D. J. F. V. and Y, C., "Infrared emissivity measurements of building and civil engineering materials: a new device for measuring emissivity," Int J Thermophys 35(9), 18171831 (2014).

[11] “Copernicus Data Store.” https://cds.climate.copernicus.eu/cdsapp\#!/home.

[12] "SMARTS: Simple Model of the Atmospheric Radiative Transfer of Sunshine — Grid Modernization NREL." https://www.nrel.gov/grid/solar-resource/smarts.html.

[13] Dumoulin, J. and Boucher, V., "Infrared thermography system for transport infrastructures survey with inline local atmospheric parameter measurements and offline model for radiation attenuation evaluations," Journal of Applied Remote Sensing 8(1), 084978-084978 (2014). 